\title{
sciendo
}

\section{ENDOVASCULAR TREATMENT FOR COMMON ILIAC AND EXTERNAL ILIAC EXTENSIVE CALCIFICATION. A CASE REPORT AND LITERATURE REVIEW}

\author{
ANDREI-FLORIN BLOJ ${ }^{1}$, LUCIAN MĂRGINEAN ${ }^{2}$, \\ CORNELIU FLORIN BUICU ${ }^{3}$, VLAD VUNVULEA ${ }^{4}$ \\ ${ }^{1,2,4}$ Emergency County Hospital, Târgu Mureş, \\ 3 “George Emil Palade” University of Medicine, Pharmacy, Science and Technology of Târgu Mureș
}

\begin{abstract}
Keywords:
atherosclerosis,

calcification, stenosis,

endovascular, angioplasty

Abstract: Peripheral artery disease (PAD) is an intricate and widespread pathology these days. Under some circumstances such as diabetes or other pathologies as comorbidities, PAD occurs much earlier in life. We report the case of a 55-year old male with diabetes mellitus (DM) and chronic kidney disease with extensive calcifications of the common and external iliac artery. After repeated balloon dilatation without success we resorted to placing two balloon mounted stents with optimal results.
\end{abstract}

\section{INTRODUCTION}

There is of common knowledge the fact that patients suffering from diabetes mellitus have a higher rate of developing atherosclerosis compared to people without DM.

Diabetes mellitus is categorized in the group of metabolic disorders with the characteristic prolonged hyperglycemia. The link between high blood sugar levels and atherosclerotic plaques is believed to be the endothelial dysfunction.(1) Usually endothelial cells maintain the homeostasis of the vascular wall by regulating inflammatory processes and anti-inflammation, coagulation and fibrinolysis or other processes like contraction and dilatation of the wall. There are a couple of well known factors that contribute to the disruption of the normal endothelial "balance", such as high cholesterol levels, hyperglycemia or oxidative stress, factors that can cause endothelial dysfunction and over time atherosclerosis.(2)

Recent studies point to local inflammation as the key factor triggering specific changes in the vascular endothelial cells involved in atherosclerosis. All these changes appear to be sped up by chronic hyperglycemia, such as in diabetes mellitus.(3)

According to the results of the latest studies (WHO) regarding the demographics of $\mathrm{DM}$, the national prevalence in Romania is $8.8 \%$, almost $1 \%$ over the European average.

Besides DM, another pathology that causes chronic endothelial inflammation is chronic kidney disease, being a major risk factor taking into account that Romania is in the top 10 list as for incidence of chronic kidney disease in the general population.(4)

Thus, the more risk factors for atherosclerosis a patient has, the more difficult the curative treatment is going to be.(5)

\section{CASE REPORT}

We present the case of a 55-year old male with diabetes mellitus since the age of 16 and chronic kidney disease undergoing dialyses since the age of 45 , accusing pain in the inferior limbs. The patient was admitted at the Târgu Mureș Emergency County Hospital for further investigation and treatment.

Figure no. 1. Initial inferior limb angiography

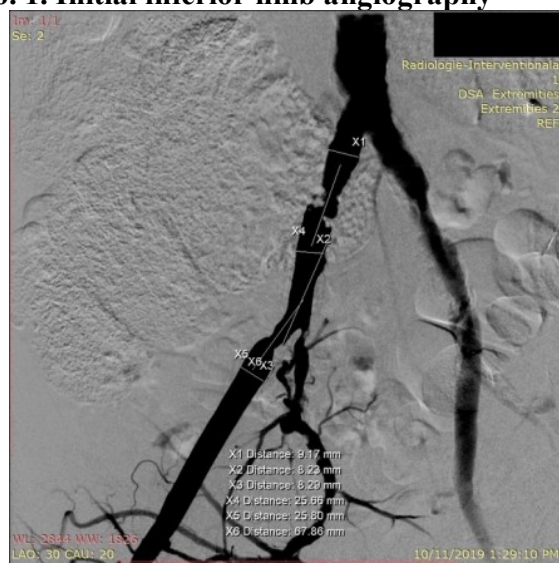

Figure no. 2. Angio CT reconstruction

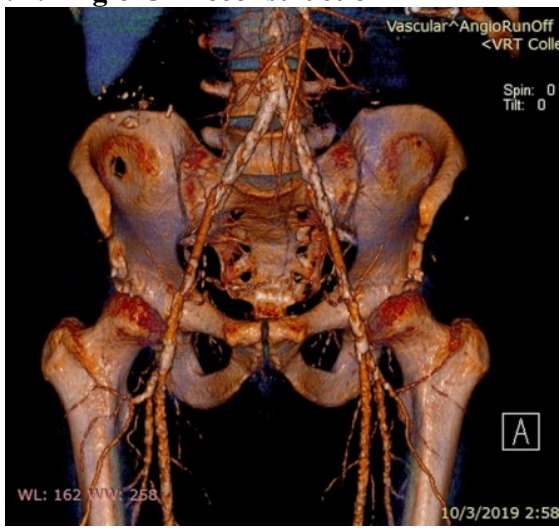

'Corresponding author: Lucian Mărginean, Str. Ghe.Marinescu, Nr. 50, Târgu-Mureş, România, E-mail: go2lucian@yahoo.com, Phone: +40744 332201

Article received on 29.07.2020 and accepted for publication on 24.08.2020 


\section{CLINICAL ASPECTS}

The initial angiography of the inferior limbs showed an irregular path of the right common and external iliac artery as shown in figures no. 1 and 2. Large calcifications covered the walls of the arteries with a length of $25.66 \mathrm{~mm}$ for the first one on the common iliac artery and a length of $25.8 \mathrm{~mm}$ for the second one on the external iliac artery, right after the bifurcation.

Using an ipsilateral retrograde approach of the right femoral artery a guide wire was introduced and we tried to dilate the vessels using a balloon stent.

After numerous attempts to inflate the balloon to pressures of almost $18 \mathrm{kPa}$ and puncturing it we were left with no other choice than to place a balloon mounted stent. Thus, we went for the Express LD iliac premounted stent system by Boston Scientific and placed the first stent on the external iliac artery (visible in figure no. 3 ) and the second one on the external iliac artery right after its bifurcation being careful to cover as little as possible the emergence of the internal iliac artery (figure no. 4).

Figure no. 3. Angiography after placing the first stent

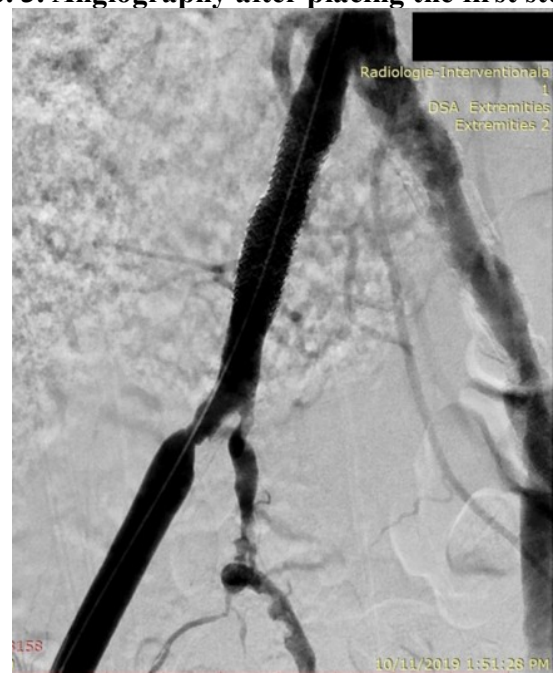

Figure no. 4. Angiography after placing the second stent

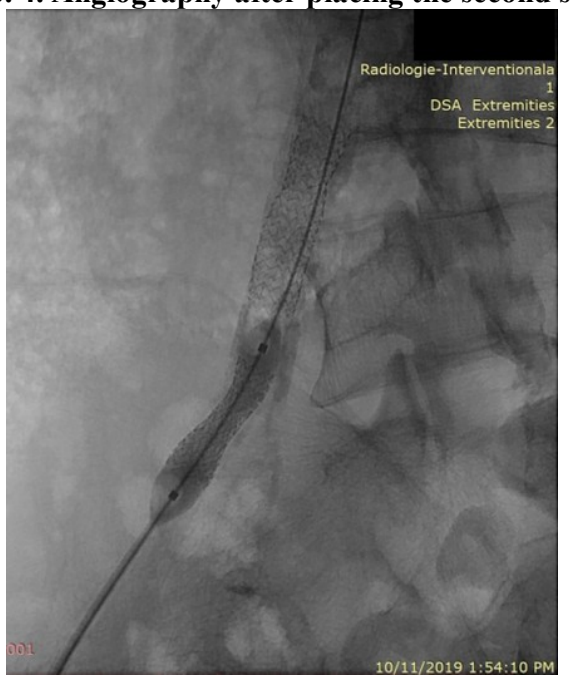

The results after revascularization were optimal with proper flow through the external iliac vessels as well as through the internal iliac artery despite the placement of the second stent as shown on figure no. 5 .

The follow-up of this patient 3 months after the intervention revealed a proper revascularization of the right inferior limb, lack of claudication or pain and full clinical recovery.

\section{Figure no. 5. Angiography after both stents were placed}

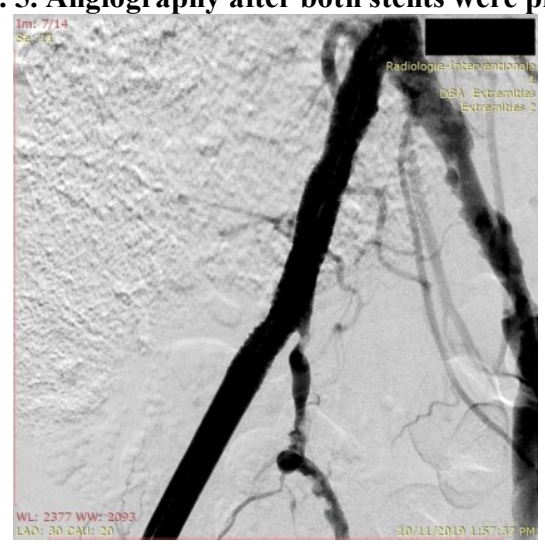

\section{DISCUSSIONS}

Peripheral arterial disease has a very high prevalence these days, thus there is a variety of techniques to treat it ranging from minimally-invasive such as endovascular treatment down to open surgery.

The TransAtlantic Inter-Society Consensus (TASC) working group published in the year 2000 a group of recommendations for the management of peripheral arterial disease.(6)

Taking into account these guidelines, by having an atherosclerotic lesion of the common iliac artery and of the external iliac artery involving the bifurcation we are looking at a TASC II C lesion where surgical intervention is recommended. However, these guidelines were introduced 20 years ago, since then we have seen a huge improvement of the endovascular techniques with great results. Recent trial studies such as CLEVER (7) show the clear superiority of endovascular treatment of iliac stenosis over other means of treatment. But the endovascular treatment is not the same for every type of lesion, there plenty varieties of approaches and techniques.

Some authors (8) highly recommend self-expandable stents for iliac lesions due to the size discrepancies between the proximal and distal stent landing zones, whereas balloon expandable stents are recommended mostly in aorto-iliac lesions.

Other authors (9) recommend balloon expandable stents as a much safer alternative for iliac lesions due to a couple of advantages such as precise landing zone and a much higher radial strength being able to overcome large calcifications of the vascular wall.

Our patient having both diabetes mellitus and chronic kidney disease, the occurrences of peripheral arterial disease in the form of large calcifications of the common and external iliac artery at the age of 55 is no surprise, but it raises a lot of discussion regarding the optimal revascularization technique in order to prevent the occurrence of complications or metabolic imbalance. The endovascular approach was optimal but we encountered difficulties when attempting the balloon dilatation due to extensive calcifications of the vascular wall, thus we resorted to balloon-stent placement with optimal results and complete recovery.

\section{CONCLUSIONS}

Nowadays, peripheral arterial disease is not necessary a pathology affecting the elderly, young patients can also develop extensive stenosis of inferior limb vessels.

Old guidelines should be updated periodically by taking into account new equipment and new techniques in 
interventional radiology.

Although our patient had large calcifications of the common and external iliac artery, balloon-stent placement had optimal results in both short- and long-term follow-up in spite his comorbidities like diabetes mellitus and chronic kidney failure.

Conflict of interests

The authors declare that they have no conflict of interests.

\section{REFERENCES}

1. Kim JA, Montagnani M, Koh KK, Quon MJ. Reciprocal Relationships Between Insulin Resistance and Endothelial Dysfunction. Molecular and Pathophysiological Mechanisms. Circulation. 2006;113:1888-1904.

2. Libby P, Ridker PM, Maseri A. Inflammation and atherosclerosis. Circulation. 2002;105:1135.

3. Lee HM, Kim JJ, Kim HJ, Shong M, Ku BJ, Jo EK.Upregulated NLRP3 inflammasome activation in patients with type 2 diabetes. Diabetes. 2013;62:194-204.

4. Stefan G, Garneata L, Tacu D, et al. Transplantation in Romania: Where Do We Stand? Maedica (Buchar). 2015;4:304+309.

5. Fowkes FG, Rudan D, Rudan I, et al. Comparison of globa estimates of prevalence and risk factors for peripheral artery disease in 2000 and 2010: a systematic review and analysis. Lancet. 2013;382:1329-40.

6. Rutherford RB. Management of peripheral arterial disease (PAD). TASC Working Group. TransAtlantic Inter-Society Concensus (TASC).J Vasc Surg. 2000;31:S1-S296.

7. Murphy TP, Cutlip DE, Regensteiner JG, et al. Supervised exercise, stent revascularization, or medical therapy for claudication due to aortoiliac peripheral artery disease: the clever study. J Am Coll Cardiol. 2015;65:999-1009. 10.1016/j.jacc.2014.12.043.

8. Kang G. Endovascular approach to iliac artery stenosis and restenosis. Indian Heart J. 2015;67(6):514-517. doi:10.1016/j.ihj.2015.09.034.

9. Cronewett Jack K., Wayne Johnston . 8th ed. 2 vol. Saunders/Elsevier; Philadelphia, PA: 2014. (Rutherford's Vascular Surgery). 